\title{
ON THE LATTICE OF SUBALGEBRAS OF AN ALGEBRA ${ }^{1}$
}

\author{
LINDA L. DENEEN
}

\begin{abstract}
Let $R$ be a Noetherian inertial coefficient ring and let $A$ be a finitely generated $R$-algebra (that is, finitely generated as an $R$-module) with Jacobson radical $J(A)$. Let $S$ be a subalgebra of $A$ with $S+J(A)=A$. We show that for every separable subalgebra $T$ of $A$ there is a unit $a$ of $A$ such that $a T a^{-1} \subseteq S$. It follows that if $S$ is separable (hence inertial) and if $T$ is a maximal separable subalgebra of $A$, then $T$ is inertial. We also show that if $S+I=A$ for a nil ideal $I$ of $A$, then $R$ can be taken to be an arbitrary commutative ring, and the conjugacy result still holds.
\end{abstract}

All rings will be associative and will possess an identity element 1 . All subrings will contain the identity of the overring, and ring homomorphisms will map the identity to the identity.

If $A$ is a ring, $R$ a commutative ring, and $\theta$ a ring homomorphism of $R$ into the center of $A$, then $\theta$ induces a natural $R$-module structure on $A$ defined by $r \cdot a=\theta(r) a$ for $r \in R, a \in A$, and we say that $A$ is an $R$-algebra. An $R$-algebra $A$ is said to be finitely generated or projective if it is finitely generated or projective as a module over $R$. For all rings $R$ we let $J(R)$ denote the Jacobson radical (or radical) of $R$. We denote by $\mu$ the multiplication map $\mu: A \otimes A^{\circ} \rightarrow A$ and let $J=\operatorname{ker} \mu$.

Recall that an $R$-algebra $A$ is separable over $R$ (or separable) if its enveloping algebra $A \otimes_{R} A^{\circ}$ contains an idempotent $e$ with the property that $\mu(e)=1$ and $J \cdot e=0$. If $A$ is a finitely generated algebra over a commutative ring $R$, then a subalgebra $S$ of $A$ is an inertial subalgebra if $S$ is a separable $R$-algebra such that $S+J(A)=A$. A commutative ring $R$ is an inertial coefficient ring if every finitely generated $R$-algebra $A$ for which $A / J(A)$ is separable contains an inertial subalgebra. Basic properties of separable algebras can be found in [3], and basic properties of inertial subalgebras and inertial coefficient rings can be found in [7].

If $A$ is a finitely generated $R$-algebra and $I$ is an ideal of $A$, then we say we can "lift idempotents from $A / I$ to $A$ " if every idempotent in $A / I$ is the image of an idempotent in $A$ under the natural map from $A$ to $A / I$. In [9, Theorem 4, p. 221] Kirkman proved that if $R$ is an inertial coefficient ring and $A$ a finitely generated $R$-algebra, then idempotents can be lifted from $A / J(A)$ to $A$. Ingraham has

Received by the editors January 30, 1982.

1980 Mathematics Subject Classification. Primary 16A16; Secondary 16A32, 16A72.

Key words and phrases. Separable algebra, inertial subalgebra, inertial coefficient ring, lifting idempotents.

${ }^{1}$ This work is part of my doctoral thesis submitted to Michigan State University. I am deeply grateful to Professor Edward C. Ingraham for directing my research. 
conjectured that the converse is true as well; that is, if $R$ has the property that idempotents can be lifted from $A / J(A)$ to $A$ for every finitely generated $R$-algebra $A$, then $R$ is an inertial coefficient ring.

Let $R$ be a commutative ring and $A$ a finitely generated $A$-algebra. We are interested in finding conditions under which a maximal separable subalgebra $T$ of $A$ is inertial. It is clear that if $S$ is an inertial subalgebra of $A$, and if $a$ is a unit of $A$ such that $a T a^{-1} \subseteq S$, then $T$ is inertial. Thus we are led to look for conditions under which we can conjugate $T$ into $S$.

We consider the more general question: Under what circumstances can any separable subalgebra $T$ of $A$ be conjugated into any subalgebra $S$ with $S+J(A)=A$ ? There are examples due to N. Ford [4] of rings $R$ and finitely generated $R$-algebras $A$ containing nonisomorphic inertial subalgebras. Since inertial subalgebras are maximal separable subalgebras [7, Theroem 2.5 , p. 80], such conjugation does not always occur. The main result of this paper is to prove that it does occur whenever $R$ is a Noetherian inertial coefficient ring.

THEOREM 1.1. Let $R$ be a Noetherian inertial coefficient ring, and let $A$ be a finitely generated $R$-algebra. Let $T$ be a separable subalgebra of $A$, and let $S$ be a subalgebra of $A$ with the property that $S+J(A)=A$. Then there is a unit a of $A$ such that $a T a^{-1} \subseteq S$.

Proof. Step 1. We first reduce to the case where $A / J(A)$ is separable over $R$. Let $A_{1}=T+J(A)$. Then $J(A) \subseteq J\left(A_{1}\right)$ [1, Corollary, p. 126], so $A_{1} / J\left(A_{1}\right)$ is a homomorphic image of $T$ and hence is separable. Setting $S_{1}=S \cap A_{1}$, we clearly have $S_{1}+J(A) \subseteq A_{1}$. To show equality, we write an arbitrary element $a_{1}$ of $A_{1}=T+$ $J(A)$ as $t+n$ where $t \in T, n \in J(A)$. Since $a_{1}$ also lies in $A=S+J(A)$, we have $t+n=s+n_{1}$ for $s \in S, n_{1} \in J(A)$. It follows that $s=t+n-n_{1}$ is in $S \cap(T+$ $J(A))=S_{1}$, so that $a_{1} \in S_{1}+J(A)$, and we have $S_{1}+J(A)=A_{1}$. If the theorem is true for $A_{1}$, then there is a unit $a \in A_{1} \subseteq A$ such that $a T a^{-1} \subseteq S_{1} \subseteq S$. Thus, it suffices to prove the theorem in the case that $A / J(A)$ is separable over $R$.

Step 2. We now reduce to the case where $S$ is separable, hence inertial. By [1, Corollary, p. 126], $S \cap J(A) \subseteq J(S)$, but $S /[S \cap J(A)] \simeq A / J(A)$ is semisimple, so $S \cap J(A)=J(S)$. Since $R$ is an inertial coefficient ring, $S$ contains a separable subalgebra $S_{1}$ such that $S_{1}+J(S)=S$, and it follows that $S_{1}+J(A)=A$. Clearly, if we can conjugate $T$ into $S_{1}$, we can conjugate it into $S$. Therefore, we assume $S$ is an inertial subalgebra.

The remainder of the proof involves the following setting. Let $\bar{A}=A / J(A)$, $\bar{T}=T /(T \cap J(A))$, and $\bar{R}=R /(R \cap J(A))$. Let $f: S \otimes_{R} T^{\circ} \rightarrow \bar{A} \otimes_{R} \bar{T}^{\circ}$ and $g:$ $T \otimes_{R} T^{\circ} \rightarrow \bar{A} \otimes_{R}-\bar{T}^{\circ}$ be the natural maps, and let $e$ be a separability idempotent for $T$ with $\bar{e}=g(e)$. Then

$$
\operatorname{ker} f=i\left[(S \cap J(A)) \otimes_{R} T^{\circ}+S \otimes_{R}(T \cap J(A))^{\circ}\right] \subseteq J\left(S \otimes_{R} T^{\circ}\right)
$$

[1, Theorem 10, p. 127]. Since $R$ is an inertial coefficient ring, idempotents can be lifted from $\left(S \otimes_{R} T^{\circ}\right) / J\left(S \otimes_{R} T^{\circ}\right)$ to $S \otimes_{R} T^{\circ}$, and it follows from [6, Corollary 1.3, p. 46] that we can lift idempotents from $\bar{A} \otimes_{R}-\bar{T}^{\circ}$ to $S \otimes_{R} T^{\circ}$, so let $e_{1}$ be an 
idempotent in $S \otimes_{R} T^{\circ}$ such that $f\left(e_{1}\right)=\bar{e}$. The picture looks like this:

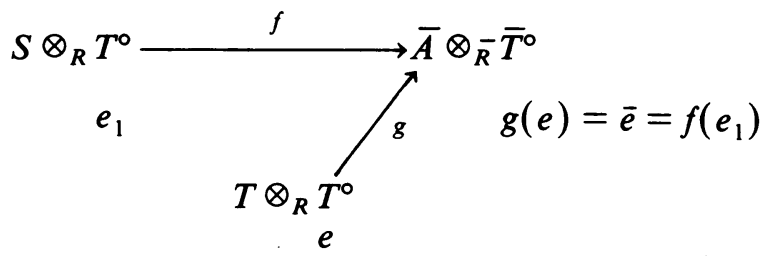

If $\mu: A \otimes_{R} A^{\circ} \rightarrow A$ is the multiplication map, then we will show that $\mu\left(e_{1}\right)$ is the conjugating element we seek. In other words, providing $e_{1}$ is an idempotent preimage of $\bar{e}$, we will show that $\mu\left(e_{1}\right) T \mu\left(e_{1}\right)^{-1} \subseteq S$.

Step 3. Let $R$ be a field. The proof of [2, Lemma 2.7, p. 127] gives the existence of a unit $\alpha$ in $A$ of the form $\alpha=1+n$ for $n$ in $J(A)$ such that $\alpha T \alpha^{-1} \subseteq S$. If we define $\varphi: T \rightarrow S$ by $\varphi(t)=\alpha t \alpha^{-1}$, then the map $\varphi \otimes 1: T \otimes_{R} T^{\circ} \rightarrow S \otimes_{R} T^{\circ}$ makes the preceding diagram commute. Furthermore,

$$
\operatorname{ker} f=i\left[(S \cap J(A)) \otimes_{R} T^{\circ}+S \otimes_{R}(T \cap J(A))^{\circ}\right]=0,
$$

since $S$ and $T$ are separable over the field $R$. Therefore, if we let $e_{1}=(\varphi \otimes 1)(e)$, then $e_{1}$ is the unique preimage of $\bar{e}$ in $S \otimes_{R} T^{\circ}$, and $e_{1}$ is also an idempotent.

Because $e$ is a separability idempotent for $T$, we have $(1 \otimes t-t \otimes 1) \cdot e=0$ for every $t$ in $T$. Applying $\varphi \otimes 1$, we have $(1 \otimes t-\varphi(t) \otimes 1) \cdot e_{1}=0$. Next apply $\mu$, recall that $\varphi(t)=\alpha t \alpha^{-1}$, and notice that $\mu\left(e_{1}\right) t-\alpha t \alpha^{-1} \mu\left(e_{1}\right)=0$. It follows that $\mu\left(e_{1}\right) t \mu\left(e_{1}\right)^{-1}=\alpha t \alpha^{-1}$ is in $S$, provided $\mu\left(e_{1}\right)$ is invertible. But $\mu(\bar{e})=1$, so $\mu\left(e_{1}\right)=$ $1+n$ for some $n \in J(A)$; consequently $\mu\left(e_{1}\right)$ is invertible.

Step 4. Suppose $(R, m)$ is a Noetherian local ring with $m^{n}=0$ for some positive integer $n$. We proceed by induction on $n$. If $n=1$, then $R$ is a field, and the result follows from Step 3. Assume the statement is true for $n \leqslant k$, and consider the case where $n=k+1$.

Let $\tilde{A}=A /\left(m^{k} A\right), \tilde{R}=R / m^{k}, \tilde{T} /\left(m^{k} A \cap T\right)$, and $\tilde{S}=S /\left(m^{k} A \cap S\right)$. Since $m^{k} A \subseteq J(A)$ by [7, Lemma 1.1, p. 78], then $J(\tilde{A})=J(A) / m^{k} A$. Letting $\tilde{e}_{1}$ and $\tilde{e}$ be the images of $e_{1}$ and $e$, and taking $\tilde{f}$ and $\tilde{g}$ to be the induced maps from $f$ and $g$, we have the following situation:

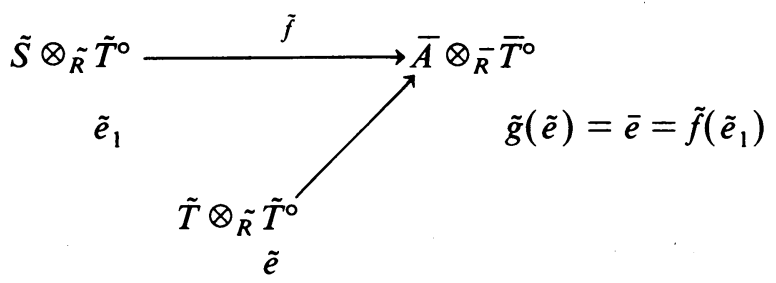

Both $\tilde{T}$ and $\tilde{S}$ are separable over $\tilde{R}, \tilde{e}$ is a separability idempotent for $\tilde{T}$, and $\tilde{S}+J(\tilde{A})=\tilde{A}$. Then the induction hypothesis gives that $\mu\left(\tilde{e}_{1}\right) \tilde{T} \mu\left(\tilde{e}_{1}\right)^{-1} \subseteq \tilde{S}$. Pulling this inclusion back to $A$, we have $\mu\left(e_{1}\right) T \mu\left(e_{1}\right)^{-1} \subseteq S+m^{k} A$.

Now let $C=S+m^{k} A$ and $T^{\prime}=\mu\left(e_{1}\right) T \mu\left(e_{1}\right)^{-1} . S$ is an inertial subalgebra of $C$, and $C$ is a finitely generated $R$-algebra because $R$ is Noetherian. Write $e=\Sigma \gamma_{i} \otimes \delta_{i}$, 
where $\gamma_{i} \in T, \delta_{i} \in T^{\circ}$, and let

$$
e^{\prime}=\sum\left[\mu\left(e_{1}\right) \gamma_{i} \mu\left(e_{1}\right)^{-1} \otimes \mu\left(e_{1}\right) \delta_{i} \mu\left(e_{1}\right)^{-1}\right] .
$$

One easily sees that $e^{\prime}$ is a separability idempotent for $T^{\prime}$. Write $e_{1}=\Sigma \alpha_{j} \otimes \beta_{j}$ where $\alpha_{j} \in S$ and $\beta_{j} \in T^{\circ}$, let $e_{1}^{\prime}=\Sigma \alpha_{j} \otimes \mu\left(e_{1}\right) \beta_{j} \mu\left(e_{1}\right)^{-1}$, and notice that $e_{1}^{\prime}$ is an idempotent. It is not hard to see that $J(C)=C \cap J(A), C / J(C)=\bar{A}$, and $T^{\prime} /\left(T^{\prime} \cap J(C)\right) \simeq \bar{T}$. Thus, we have natural maps $f^{\prime}: S \otimes_{R} T^{\circ \circ} \rightarrow \bar{A} \otimes_{R}-\bar{T}^{\circ}$ and $g^{\prime}:$ $T^{\prime} \otimes_{R} T^{\prime \circ} \rightarrow \bar{A} \otimes_{R}-\bar{T}^{\circ}$ with $f^{\prime}\left(e^{\prime}{ }_{1}\right)=\bar{e}=g^{\prime}\left(e^{\prime}\right)$. We can now use the same argument here for $C$ that we used previously for $A$ to conclude that

$$
\mu\left(e_{1}^{\prime}\right) T^{\prime} \mu\left(e_{1}^{\prime}\right)^{-1} \subseteq S+m^{k} C=S+m^{k}\left(S+m^{k} A\right)=S .
$$

Equivalently, $\mu\left(e_{1}^{\prime}\right) \mu\left(e_{1}\right) T \mu\left(e_{1}\right)^{-1} \mu\left(e_{1}^{\prime}\right)^{-1} \subseteq S$. But

$$
\begin{aligned}
\mu\left(e_{1}^{\prime}\right) \mu\left(e_{1}\right) & =\left(\sum \alpha_{j} \mu\left(e_{1}\right) \beta_{j} \mu\left(e_{1}\right)^{-1}\right) \cdot \mu\left(e_{1}\right)=\sum \alpha_{j} \mu\left(e_{1}\right) \beta_{j} \\
& =\left[\sum \alpha_{j} \otimes \beta_{j}\right] \cdot \mu\left(e_{1}\right)=e_{1} \cdot \mu\left(e_{1}\right)=\mu\left(e_{1} \cdot e_{1}\right)=\mu\left(e_{1}\right) .
\end{aligned}
$$

Thus we have shown that $\mu\left(e_{1}\right) T \mu\left(e_{1}\right)^{-1} \subseteq S$.

Step 5. Let $(R, m)$ be a Noetherian local ring. Let $k$ be a positive integer, and pass to the factor algebra $\tilde{A}=A / m^{k} A$ over $\tilde{R}=R / m^{k}$. Letting $\tilde{T}=T /\left(m^{k} A \cap T\right)$ and $\tilde{S}=S /\left(m^{k} A \cap S\right)$, we have that $\tilde{T}$ is $\tilde{R}$-separable and $\tilde{S}$ is an $\tilde{R}$-inertial subalgebra of $\tilde{A}$. Defining $\tilde{e}, \tilde{e}_{1}, \tilde{f}$, and $\tilde{g}$ as in Step 4 , we apply the result of Step 4 to get $\mu\left(\tilde{e}_{1}\right) \tilde{T} \mu\left(\tilde{e}_{1}\right)^{-1} \subseteq \tilde{S}$. Pulling back to $A$ we have $\mu\left(e_{1}\right) T \mu\left(e_{1}\right)^{-1} \subseteq S+m^{k} A$. The containment holds for every positive integer $k$, so $\mu\left(e_{1}\right) T \mu\left(e_{1}\right)^{-1} \subseteq \cap_{k=1}^{\infty}\left(S+m^{k} A\right)$. But $R$ is a Zariski ring [11, pp. 263-264], so $\cap_{k=1}^{\infty}\left(S+m^{k} A\right)=S$, and again we have shown that $\mu\left(e_{1}\right) T \mu\left(e_{1}\right)^{-1} \subseteq S$.

Step 6. Let $R$ be a Noetherian ring and $T^{\prime}=\mu\left(e_{1}\right) T \mu\left(e_{1}\right)^{-1}$. We will show that $T^{\prime} \subseteq S$ by showing that $Z=\left(T^{\prime}+S\right) / S$ is the zero module. $Z=0$ if and only if $Z_{m}=Z \otimes_{R} R_{m}=0$ for every maximal ideal $m$ of $R$ [3, Proposition 4.4, p. 29]. By tensoring everything in the diagram in Step 2 with $R_{m}$ over $R$, we again place ourselves in the setting of Step 5 , where we have $T_{m}^{\prime} \subseteq S_{m}$, or equivalently, $Z_{m}=0$. We conclude that $Z=0$, and it follows that $\mu\left(e_{1}\right) T \mu\left(e_{1}\right)^{-1} \subseteq S$.

EXAMPLE. Let $R=Z / 4 Z, A=M_{2}\left[R[x] /\left(x^{2}-2\right)\right]$, and $S=M_{2}(R)$. Then $J(A)$ $=A\left[\begin{array}{l}\bar{x} \\ 0\end{array}\right]+2 A$, so $S$ is an inertial subalgebra of $A$. Moreover,

$$
T=\left\{\left[\begin{array}{cc}
3 a+2 b & (a+3 b) \bar{x} \\
(3 a+b) \bar{x} & 2 a+3 b
\end{array}\right] a, b \in R\right\}
$$

is a separable subalgebra of $A$ with separability idempotent

$$
e=\left[\begin{array}{cc}
3 & \bar{x} \\
3 \bar{x} & 2
\end{array}\right] \otimes\left[\begin{array}{cc}
3 & \bar{x} \\
3 \bar{x} & 2
\end{array}\right]+\left[\begin{array}{cc}
2 & 3 \bar{x} \\
\bar{x} & 3
\end{array}\right] \otimes\left[\begin{array}{cc}
2 & 3 \bar{x} \\
\bar{x} & 3
\end{array}\right]
$$

Let

$$
z=\left[\begin{array}{ll}
3 & 2 \\
2 & 2
\end{array}\right] \otimes\left[\begin{array}{cc}
3 & \bar{x} \\
3 \bar{x} & 2
\end{array}\right]+\left[\begin{array}{ll}
2 & 2 \\
2 & 1
\end{array}\right] \otimes\left[\begin{array}{cc}
2 & 3 \bar{x} \\
\bar{x} & 3
\end{array}\right]
$$


where $z \in S \otimes_{R} T^{\circ}$ and $f(z)=\bar{e}=g(e)$. Notice that $z$ is not an idempotent, and furthermore, that $\mu(z) T \mu(z)^{-1} \nsubseteq S$. Thus we see that there are nonidempotent preimages $z$ of $\bar{e}$ in $S \otimes_{R} T^{\circ}$ for which $\mu(z)$ does not conjugate $T$ into $S$.

In the proof of Theorem 1.1, once the reductions of Steps 1 and 2 are made, the only place we use that $R$ is an inertial coefficient ring is when we wish to lift idempotents. Thus, if we start with the assumptions that $A / J(A)$ is separable and $S$ is an inertial subalgebra of $A$, we have the following corollary.

COROLlaRY 1.2. Let $R$ be a Noetherian ring with the property that for every finitely generated $R$-algebra, idempotents can be lifted from the algebra modulo its radical to the algebra. Let $A$ be a finitely generated $R$-algebra with $A / J(A)$ separable, let $S$ be an inertial subalgebra of $A$, and let $T$ be a separable subalgebra of $A$. Then there is a unit a in $A$ with $a T a^{-1} \subseteq S$.

Conjugates of inertial subalgebras are inertial subalgebras, so if we are in a setting where inertial subalgebras exist and Theorem 1.1 applies, the following corollary holds.

Corollary 1.3. If $R$ is a Noetherian inertial coefficient ring and $A$ is a finitely generated $R$-algebra with $A / J(A)$ separable, then every separable subalgebra is contained in an inertial subalgebra, and every maximal separable subalgebra is an inertial subalgebra.

When $A$ is a commutative, finitely generated algebra over a commutative ring $R$, the situation becomes much simpler.

PROPOSITION 1.4. Let $A$ be a commutative, finitely generated algebra over $a$ commutative ring $R$. Let $S$ be a subalgebra of $A$ with $S+J(A)=A$. If $T$ is $a$ separable subalgebra of $A$, then $T \subseteq S$.

Proof. If we consider $A$ as an $S$-algebra, then $S$ is an $S$-inertial subalgebra of $A$. Then $S \otimes_{R} T$ is $S$-separable, and $S \cdot T$ is a homomorphic image of $S \otimes_{R} T$, so $S \cdot T$ is an $S$-separable subalgebra of $A$. Furthermore, since $S \subseteq S \cdot T, S \cdot T$ is also an $S$-inertial subalgebra of $A$. Therefore, by [7, Proposition 2.6, p. 80], $S \cdot T=S$, and consequently $T \subseteq S$.

The following result removes both the Noetherian and inertial coefficient ring conditions on $R$ in Theorem 1.1, but we are forced to replace the Jacobson radical of $A$ with a nil ideal $I$ of $A$. Ford's example shows that we cannot expect this result to be true for $J(A)$ instead of $I$. It is still unknown whether Theorem 1.1 is true when $R$ is an inertial coefficient ring which is not necessarily Noetherian.

Proposition 1.5. Let $R$ be a commutative ring and $A$ be a finitely generated $R$-algebra. Let $I$ be any nil ideal of $A$, and let $S$ be an $R$-subalgebra of $A$ such that $S+I=A$. If $T$ is any separable subalgebra of $A$, then there exists an element $a$ in $A$ such that $a T a^{-1} \subseteq S$.

Proof. We use the technique of selecting a suitable Hilbert subring $R_{1}$ of $R$ and an $R_{1}$-algebra $A_{1}$ which satisfy the conditions of Theorem 1.1. We then lift the result back to $A$. 
By [10, Theorem 5, p. 5], $T$ is a finitely generated $R$-algebra, so write $T=R t_{1}+$ $R t_{2}+\cdots+R t_{m}$ for $t_{i} \in T$. $T$ is $R$-separable so there are elements $x_{i}$ and $y_{i}$ in $T$ such that $\sum x_{i} \otimes y_{i}$ is a separability idempotent for $T$ in $T \otimes_{R} T^{\circ}$. Thus we have, for every $j=1, \ldots, m$,

$$
\left(t_{j} \otimes 1-1 \otimes t_{j}\right)\left(\sum x_{i} \otimes y_{i}\right)=0 \quad \text { in } T \otimes_{R} T^{\circ}
$$

Think of $T \otimes_{R} T^{\circ}$ as a free abelian group with subgroup $\mathbb{Q}$ of relations factored out, and notice that there is a finite subset $M_{j}$ of $T \cup R$ such that the elements of $\mathscr{Q}$ making (*) zero in $T \otimes_{R} T^{\circ}$ are expressible in terms of the elements of $M_{j}$.

Let $a_{1}, \ldots, a_{n}$ generate $A$ as an $R$-module. Since $S+I=A$, there exist $s_{1}, \ldots, s_{n}$ in $S$ and $\mu_{1}, \ldots, \mu_{n}$ in $I$ with $a_{i}=s_{i}+\mu_{i}$ for $i=1, \ldots, n$.

Now set $B=\left\{1, a_{i} a_{j}, s_{i}, t_{i}\right\}$ and $C=\left\{1, t_{i} t_{j}, x_{i}, y_{i}\right\} \cap\left(\cup_{j} M_{j}\right)$. Write each element of the finite set $B$ as an $R$-linear combination of $a_{1}, \ldots, a_{n}$, and write each element of the finite set $C$ as an $R$-linear combination of $t_{1}, \ldots, t_{m}$. All of this will involve only finitely many coefficients from $R$. Let $R_{1}$ be the Noetherian subring of $R$ generated by this finite set and the "prime" subring $P$ of $R . P$ is a homomorphic image of the Hilbert ring $Z$, the integers, so $P$ is a Hilbert ring. $R_{1}$ is finitely generated as an algebra over $P$, so $R_{1}$ is a Hilbert ring [5, Theorems 2 and 3, pp. 136-137]. Therefore, $R_{1}$ is an inertial coefficient ring [8, Corollary 2, p. 553].

Define $A_{1}=R_{1} a_{1}+R_{1} a_{2}+\cdots+R_{1} a_{n}$. By construction of $R_{1}$, we have $B \subseteq A_{1}$, so $A_{1}$ is a finitely generated $R_{1}$-algebra containing the $s_{i}$ 's and the $t_{i}$ 's. Consequently, we can take $S_{1}$ to be the $R_{1}$-subalgebra of $A_{1}$ generated by $s_{1}, \ldots, s_{n}$. Next let $T_{1}=R_{1} t_{1}+R_{1} t_{2}+\cdots+R_{1} t_{m}$, so $T_{1}$ is a finitely generated $R_{1}$-algebra containing the set $C$. Furthermore, $\sum x_{i} \otimes y_{i}$ is an element of $T_{1} \otimes_{R_{1}} T_{1}^{\circ}$ satisfying (*) in $T_{1} \otimes_{R_{1}} T_{1}^{\circ}$. Consequently, $\sum x_{i} \otimes y_{i}$ is a separability idempotent for $T_{1}$, so $T_{1}$ is $R_{1}$-separable. Finally, we let $I_{1}=I \cap A_{1}$. Since $I$ is nil, $I_{1}$ is nil, and it follows that $I_{1} \subseteq J\left(A_{1}\right)$. Recall that $a_{i}-s_{i}=\mu_{i}$ is in $I$, and since $a_{i}-s_{i}$ is also in $A_{1}$, then $a_{i}-s_{i}=\mu_{i}$ is in $I_{1}$ for $i=1,2, \ldots, n$. The relations $a_{i}=s_{i}+\mu_{i}$ imply that $S_{1}+I_{1}$ $=A_{1}$, and it follows that $S_{1}+J\left(A_{1}\right)=A_{1}$.

Now $A_{1}$ satisfies all the conditions of Theorem 1.1, so there is a unit $a$ in $A_{1}$ such that $a T_{1} a^{-1} \subseteq S_{1}$. We extend back up to $A$ by multiplying by $R$ to get $R A_{1}=A$, $R S_{1} \subseteq S$, and $R T_{1}=T$. Consider $a$ now as an element of $A$, we have $a T a^{-1}=$ $a\left(R T_{1}\right) a^{-1}=R\left(a T_{1} a^{-1}\right) \subseteq R S_{1} \subseteq S$, and we are done.

Note. If $R$ is a Hilbert ring, the hypothesis of Proposition 1.5 are fulfilled for $I=J(A)$.

\section{REFERENCES}

1. G. Azumaya, On maximally central algebras, Nagoya Math. J. 2 (1951), 119-150.

2. J. Bogart, Wedderburn specters and the structure of certain associative algebras, J. Algebra 50 (1978), $122-134$.

3. F. DeMeyer and E. Ingraham, Separable algebras over commutative rings, Lecture Notes in Math., vol. 181, Springer-Verlag, Berlin and New York, 1971.

4. N. Ford, Inertial subalgebras of central separable algebras, Proc. Amer. Math. Soc. 60 (1976), 39-44.

5. O. Goldman, Hilbert rings and the Hilbert Nullstellensatz, Math. Z. 54 (1951), 136-140.

6. S. Greco, Algebras over nonlocal Hensel rings, J. Algebra 8 (1968), 45-59. 
7. E. Ingraham, Inertial subalgebras of algebras over commutative rings, Trans. Amer. Math. Soc. 124 (1966), 77-93.

8.

9. E. Kirkman, Inertial coefficient rings and the idempotent lifting property, Proc. Amer. Math. Soc. 61 (1976), 217-222.

10. D. Sanders, The dominion and separable subalgebras of finitely generated algebras, Proc. Amer. Math. Soc. 48 (1975), 1-7.

11. O. Zariski and P. Samuel, Commutative algebra. II, Van Nostrand, Princeton, N.J., 1960.

Department of Mathematics, Michigan State University, East Lansing, Michigan 48824

Current address: Department of Mathematics, Beloit College, Beloit, Wisconsin 53511 